\title{
Independent degeneration of photoreceptors and retinal pigment epithelium in conditional knockout mouse models of choroideremia
}

\author{
Tanya Tolmachova, ${ }^{1}$ Ross Anders, ${ }^{1}$ Magnus Abrink, ${ }^{2}$ Laurence Bugeon, ${ }^{3}$ Margaret J. Dallman, ${ }^{3}$ \\ Clare E. Futter, ${ }^{4}$ José S. Ramalho, ${ }^{5}$ Felix Tonagel, ${ }^{6}$ Naoyuki Tanimoto, ${ }^{6}$ Mathias W. Seeliger, ${ }^{6}$ \\ Clare Huxley, ${ }^{1}$ and Miguel C. Seabra ${ }^{1}$

\begin{abstract}
${ }^{1}$ Molecular and Cellular Medicine Section, Division of Biomedical Sciences, Imperial College London, London, United Kingdom. 2Department of Molecular Biosciences, Swedish University of Agricultural Sciences, Uppsala, Sweden. ${ }^{3}$ Section of Immunology and Infection, Department of Biological Sciences, Imperial College London, London, United Kingdom. ${ }^{4}$ nstitute of Ophthalmology, University College London, London, United Kingdom. ${ }^{5}$ Centre of Ophthalmology, Biomedical Institute for Research in Light and Image, University of Coimbra, Coimbra, Portugal.
\end{abstract} \\ ${ }^{6}$ Retinal Diagnostics Research Group, University Eye Hospital, Department II, Tuebingen, Germany.
}

\begin{abstract}
Choroideremia (CHM) is an X-linked degeneration of the retinal pigment epithelium (RPE), photoreceptors, and choroid, caused by loss of function of the CHM/REP1 gene. REP1 is involved in lipid modification (prenylation) of Rab GTPases, key regulators of intracellular vesicular transport and organelle dynamics. To study the pathogenesis of CHM and to develop a model for assessing gene therapy, we have created a conditional mouse knockout of the Chm gene. Heterozygous-null females exhibit characteristic hallmarks of CHM: progressive degeneration of the photoreceptors, patchy depigmentation of the RPE, and Rab prenylation defects. Using tamoxifen-inducible and tissue-specific Cre expression in combination with floxed Chm alleles, we show that CHM pathogenesis involves independently triggered degeneration of photoreceptors and the RPE, associated with different subsets of defective Rabs.
\end{abstract}

\section{Introduction}

Inherited retinal degenerations are a significant cause of morbidity in the Western world. Unraveling the underlying genes and their functions has led to much progress in assessing and treating these conditions and, in addition, has contributed significantly to our understanding of retinal degenerative mechanisms, including those underlying common conditions such as age-related macular degeneration (1-3). One such genetic disease is choroideremia (CHM) (OMIM 303100) (4-6). CHM has an incidence of 1 in 50,000 and exhibits X-linked inheritance. Affected males develop night blindness in their teens, which progresses to loss of peripheral vision and complete blindness $2-3$ decades after onset. The majority of heterozygous females usually do not develop blindness, although examination of the fundus often reveals a patchy distribution of abnormal areas and there is a wide range of severity due to random $\mathrm{X}$-inactivation.

Loss-of-function mutations in the CHM gene underlie CHM (7, 8). The CHM gene was renamed Rab escort protein-1 (REP1) given its role as a regulator of Rab GTPases (9). Rabs are monomeric GTPases, which function as regulators of intracellular vesicular transport and organelle dynamics $(10,11)$. To bind to intracellular membranes, Rabs have to undergo lipid modification with geranylgeranyl groups, a process generally known as prenylation (12, 13). REP binds a newly synthesized Rab, presents it to Rab geranylgeranyl transferase, which catalyzes geranylgeranyl additions to $\mathrm{Rab}$, and then "escorts" and delivers it to its target cellular mem-

Nonstandard abbreviations used: CHM, choroideremia; ERG, electroretinogram; ONL, outer nuclear layer; REP, Rab escort protein; ROS, rod outer segment; RPE, retinal pigment epithelium; SLO, scanning-laser ophthalmoscopy; TM, tamoxifen.

Conflict of interest: The authors have declared that no conflict of interest exists. Citation for this article: J. Clin. Invest. 116:386-394 (2006). doi:10.1172/JCI26617. brane (14). More than 60 Rabs have been identified in the human genome, of which some are expressed ubiquitously and others in specialized cell types, depending on the specific vesicular transport pathway they regulate $(11,15)$.

In CHM, the loss of REP1 is apparently largely compensated for by REP2, and both REPs are ubiquitously expressed (16). Intriguingly, however, the loss of REP1 is not fully compensated for in the eye, and this leads to the slow-onset retinal degeneration characteristic of CHM (17). Our previous studies suggested that the molecular basis of CHM resides in the existence of some Rabs that are selectively underprenylated in the absence of REP1. One such candidate is Rab27a, which was found to be underprenylated in lymphoblasts of CHM patients $(17,18)$.

The pathogenesis of CHM remains unclear. The name reflects the fact that the choroid is severely affected, especially at the late stages of the disease. However, 2 other layers of the eye are also damaged: the retinal pigment epithelium (RPE) and the photoreceptors. It is not known at present whether the 3 affected layers, photoreceptors, RPE, and choroid, degenerate independently or in a cascade mechanism, and, if the latter is true, which layer degenerates first.

A previous attempt to generate a $\mathrm{KO}$ mouse model of $\mathrm{CHM}$ showed, surprisingly, that null mutations of the X-linked Chm gene are embryonically lethal in males and also in heterozygous females when the mutant allele is inherited from a heterozygous female (19). The lethality is due to defects in trophoblast development and vascularization (20). In heterozygous female offspring, this is presumably due to the preferential inactivation of the paternal (wild-type) X chromosome in murine extraembryonic tissues, resulting in expression of the maternal (mutant) copy $(19,20)$. Recently, a chm knockout in zebrafish was generated by random mutagenesis. The results were similar to those in mice: chm loss 


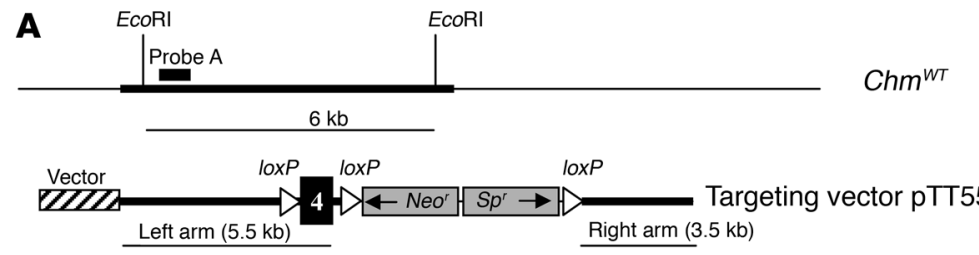

B
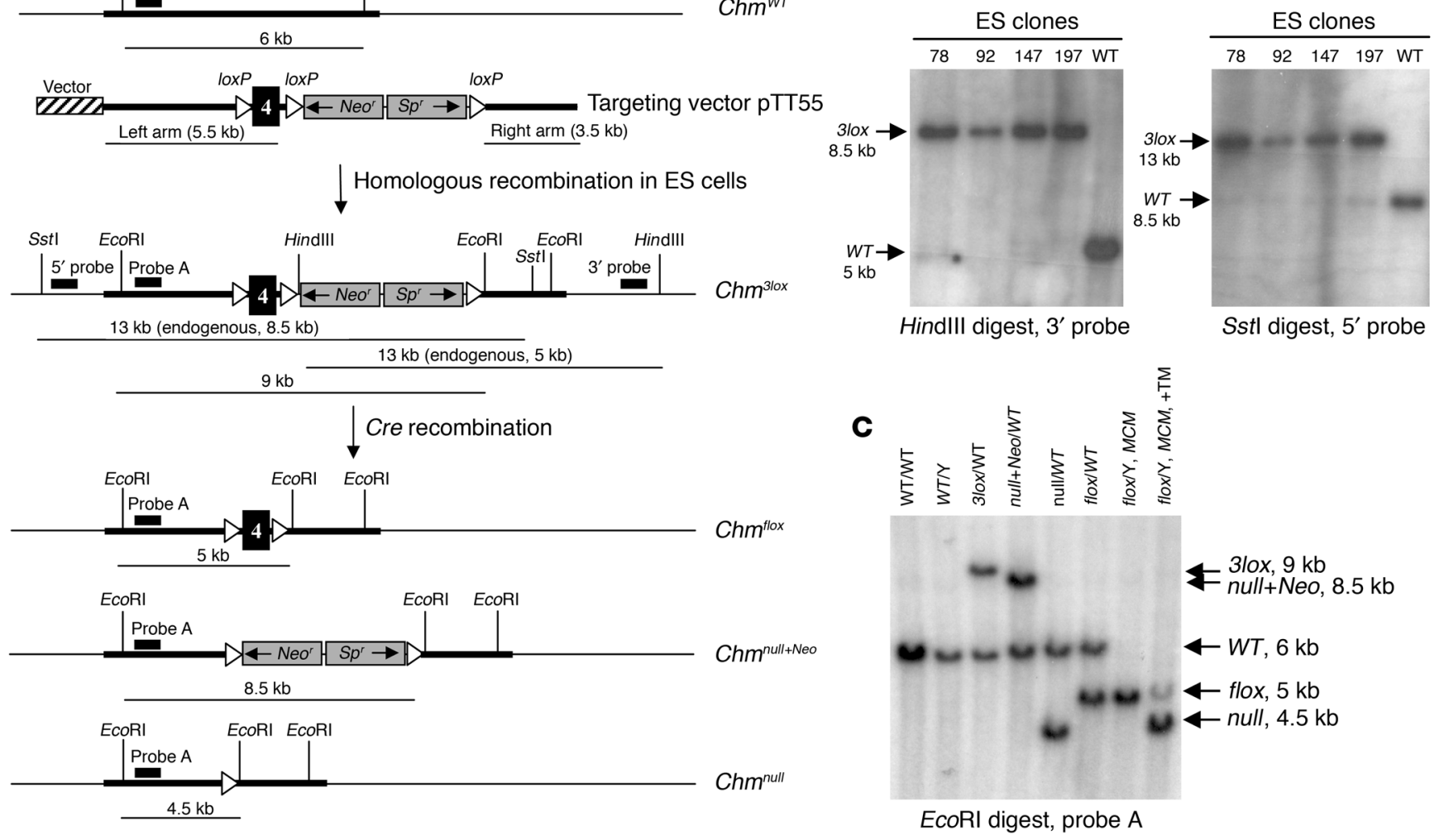

Figure 1

Generation of mice carrying the conditional and KO Chm alleles. (A) Targeting vector pTT55 carrying 3 loxP sites, a neomycin and spectinomycin resistance cassette $\left(\mathrm{NeO}^{r}, \mathrm{Sp}^{r}\right)$, and 2 homology arms was used to generate the $\mathrm{Chm}^{3 / 0 x}$ allele in GSI-1 ES cells by homologous recombination. Diagnostic HindIII and Sstl restriction sites and corresponding $3^{\prime}$ and $5^{\prime}$ probes were used to identify correctly targeted ES clones. Cre-mediated recombination between the 3 loxP sites within the $\mathrm{Chm}^{3 l o x}$ allele resulted in 3 possible alleles: $\mathrm{Chm}^{\text {flox }}, \mathrm{Chm}^{\text {null+Neo }}$, and $\mathrm{Chm}^{\text {null }}$, which were distinguished by Southern blot analysis using EcoRI digestion and probe A. (B) Results of Southern blot analysis using Hindlll digestion and the 3' probe, and Sstl digestion and the 5' probe, are shown for 4 correctly targeted ES clones $(78,92,147$, and 197) and a wild-type ES clone. (C) Results of Southern blot analysis using EcoRI digestion and probe A. The Chm alleles of the mice are indicated above the lanes. "MCM" indicates mice carrying the MerCreMer transgene. "+TM" indicates treatment with TM.

of function caused lethality of the mutant larvae, which died at 6 days after fertilization (21).

In this work, we report the generation of several mouse models of CHM. To avoid embryonic lethality and to circumvent the breeding problems caused by the inability to transmit the Chm ${ }^{\text {null }}$ allele from carrier females, we used a conditional approach in which Cre/ loxP site-specific recombination allows spatial and temporal control of the actual knockout event. The biochemical, histological, and functional analysis of these mice suggests a cell-autonomous degeneration model for this disease and reveals novel insights into retinal degenerations in general.

\section{Results}

Generation of mice with Chm-null alleles. The targeting vector PTT55 was used to generate mice carrying the $\mathrm{Chm}^{3 l o x}$ allele (Figure $1, \mathrm{~A}-\mathrm{C}$ ). The pTT55 vector contains exons 3 and 4 of the murine Chm gene within a 9-kb fragment of genomic DNA, including a $3.5-\mathrm{kb}$ right homology arm and a 5.5-kb left homology arm (Figure 1A). A neomycin and spectinomycin resistance cassette flanked by 2 loxP sites was positioned 90 bp downstream from the $3^{\prime}$ end of exon 4 . A third loxP site was inserted $280 \mathrm{bp}$ upstream from the $5^{\prime}$ end of exon 4 within the left homology arm. This allows conditional deletion of exon 4, which causes a frameshift mutation and appearance of an early stop codon.

$\mathrm{Chm}^{3 l o x}$ mice were mated with the mouse line $\beta \mathrm{MCM} 70$ (see Methods for more details), which expresses Cre as a fusion protein with 2 copies of a tamoxifen-responsive (TM-responsive) estrogen receptor (MerCreMer) under control of the ubiquitous CMVenhanced chicken $\beta$-actin promoter pCAGGS (22). MerCreMer is normally sequestered by Hsp90 in the cytoplasm. Binding to TM induces nuclear translocation of MerCreMer, which then catalyzes recombination between loxP sites. We first obtained $\mathrm{MerCreMer}^{+}$ $\mathrm{Chm}^{3 l o x} / \mathrm{Y}$ males and treated them with TM, which induced germline recombination of the Chm floxed allele. TM-treated MerCre$\mathrm{Mer}^{+} \mathrm{Chm}^{3 l o x} / \mathrm{Y}$ males were mated with wild-type females, and the female offspring carried a $\mathrm{Chm} m^{W T}$ allele from the mother and either $\mathrm{Chm}^{3 l o x}$ or 1 of the 3 possible deleted alleles, Chm ${ }^{\text {null }+\mathrm{Neo}}, \mathrm{Chm}^{\text {mull }}$, or $\mathrm{Chm}^{\text {flox }}$, from the father (Figure 1, A and C). Chm null +Neo and Chm null are null alleles, while $\mathrm{Chm}^{\text {flox }}$ is similar to the wild type, except for the presence of 2 loxP sites on either side of exon 4. This breeding scheme produced an ample supply of heterozygous-null females and thus overcame the breeding problems caused by lethality of 
A
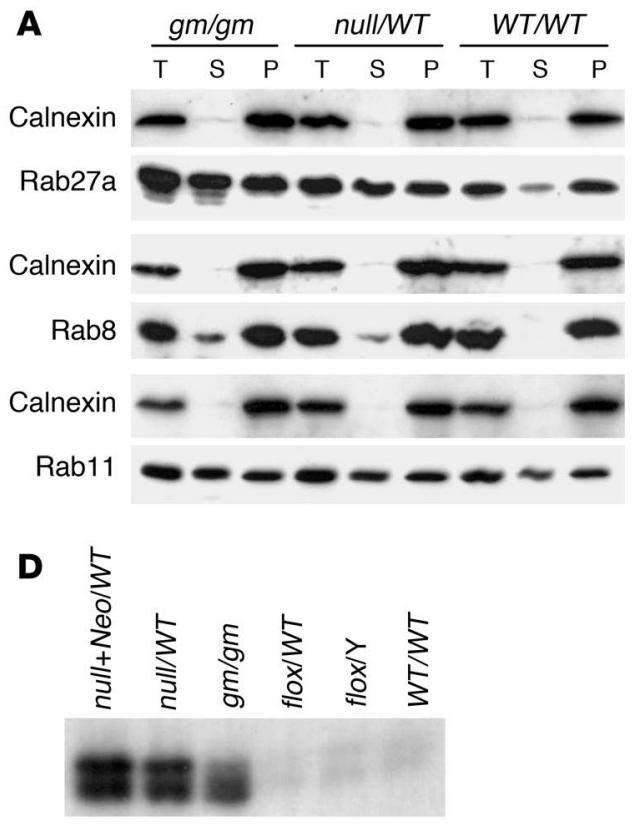

B
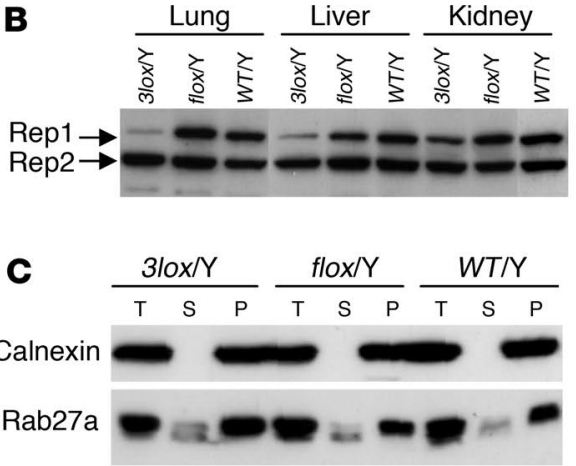

$\mathbf{E}$

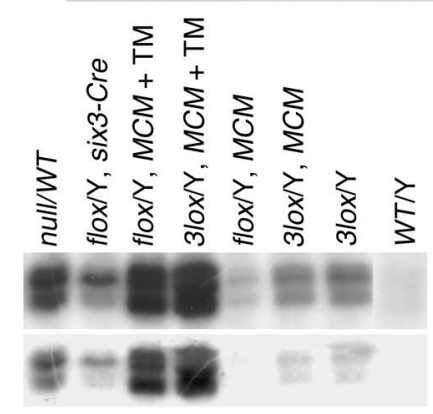

Figure 2

Analysis of Rep1 protein amount and function. (A) Total protein lysates from stomach of gunmetal $(\mathrm{gm} / \mathrm{gm}), \mathrm{Chm}^{\text {null/WT}}$, and $\mathrm{Chm} \mathrm{m}^{\text {WT/WT}}$ mice were subjected to subcellular fractionation, and total $(\mathrm{T})$, soluble $(S)$, and pellet $(P)$ fractions were immunoblotted and probed with anti-Rab27a antibody 4B12, antiRab8 antibody, and anti-Rab11 antibody as indicated. Calnexin was used as a control for subcellular fractionation. (B) Immunoblot of total protein lysates from lung, liver, and kidney of $C h m^{3 / 0 x} / Y, C h m^{f l o x} / Y$,

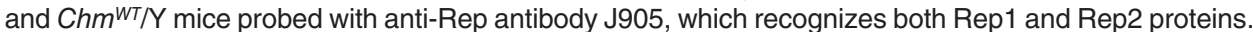
(C) Total protein lysates from large intestines of $\mathrm{Chm}^{3 / 0 x} / \mathrm{Y}, \mathrm{Chm}^{f l o x} / \mathrm{Y}$, and $\mathrm{Chm}^{\mathrm{WT}} / \mathrm{Y}$ mice were subjected to subcellular fractionation, and total $(T)$, soluble $(S)$, and pellet $(P)$ fractions were immunoblotted and probed with anti-Rab27a antibody 4B12. Calnexin was used as a control for subcellular fractionation. (D and E) In vitro prenylation was carried out on the soluble fraction of total protein lysates from large intestines (D) and eyes (E). The Chm alleles are indicated above the lanes. "MCM" indicates mice carrying the MerCreMer transgene. "+TM" indicates treatment with TM. The radiogram underneath shows a shorter exposure to film.

the Chm-null alleles in males and in females when inherited from the mother (19), a result confirmed in the present study. As Chm is $\mathrm{X}$-linked, heterozygous-null females are mosaic for cells with normal Rep 1 function or no Rep 1 function. Approximately $50 \%$ of the cells are expected to be $\mathrm{Cbm} \mathrm{KO}$ cells, thus allowing analysis of Rep1 deficiency. The ratio between normal and Chm KO cells may vary in individual mice because of random $\mathrm{X}$-inactivation.

Loss of Rep1 function causes a defect in prenylation of Rab27a. We showed previously that there is a selective defect in Rab prenylation in lymphoblast cells from CHM patients, namely in Rab27a (18). Therefore, we analyzed the prenylation status of Rab27a in mice with the engineered $\mathrm{Cbm}$ alleles by subcellular fractionation and subsequent immunoblot analysis (Figure 2A). In wild-type mice Rab27a was predominantly in the pellet fraction (Figure 2A), while in $C h m^{\text {null/WT}}$ mice approximately $50 \%$ of the protein was in the cytosolic fraction (as expected for heterozygous mice). Similar results were obtained for $\mathrm{Cbm}^{\text {mull }+ \text { Noo } / W T}$ mice (data not shown). These data confirm the requirement for Rep1 in prenylation of Rab27a and the loss of function in the null alleles (18). Loss of membrane partitioning of Rab27a was also observed for gunmetal mice, which exhibit an $80 \%$ reduction in Rab geranylgeranyl transferase activity leading to a defect in Rab prenylation and were used here as a in Chm null/WT -derived cytosolic extracts, radiolabeled $\left(\left[{ }^{3} \mathrm{H}\right]\right.$-geranylgeranylated) Rabs were observed after in vitro prenylation (Figure 2D), consistent with previous observations in human CHM cells (18). No radiolabeled Rabs were observed in $\mathrm{Cbm}$ flox $^{\text {I }} \mathrm{Y}$ extracts, but a small amount was observed in $\mathrm{Chm}^{3 l o x} / \mathrm{Y}$ extracts (Figure 2E), indicating that approximately $10 \%$ of normal Rep 1 function is not enough to ensure completely normal prenylation.

Ophthalmoscopic and electroretinographic analysis reveals pathology characteristic of CHM. We analyzed $C b m^{\text {null } / W T}$ and $C h m^{\text {null }+N e o / W T}$ mice at the ages of 1, 2, 4, 6, 8, and 10 months by indirect ophthalmoscopy. In these mice, we observed an early onset and progressive retinopathy (see Supplemental Figure 1; supplemental material available online with this article; doi:10.1172/JCI26617DS1). The fundus was characterized by hypopigmentation observed throughout the whole retina, presumably reflecting areas of focal thinning of the neuroretina and RPE exposing the choroid. Small whitish patches were apparent on ophthalmoscopic examination of 1-month-old animals, and, as the disease progressed, these areas became confluent and islands of intact retina were noted. The fundi of $C h m^{30 x} / Y$, $C h m^{3 l o x} / W T, C b m^{f l o x} / Y, C b m^{f l o x} / W T$, and $C h m^{W T} / Y$ mice were normal.

Scanning-laser ophthalmoscopy (SLO) analysis of heterozygousnull females showed an early onset and progressive retinopathy. 
A
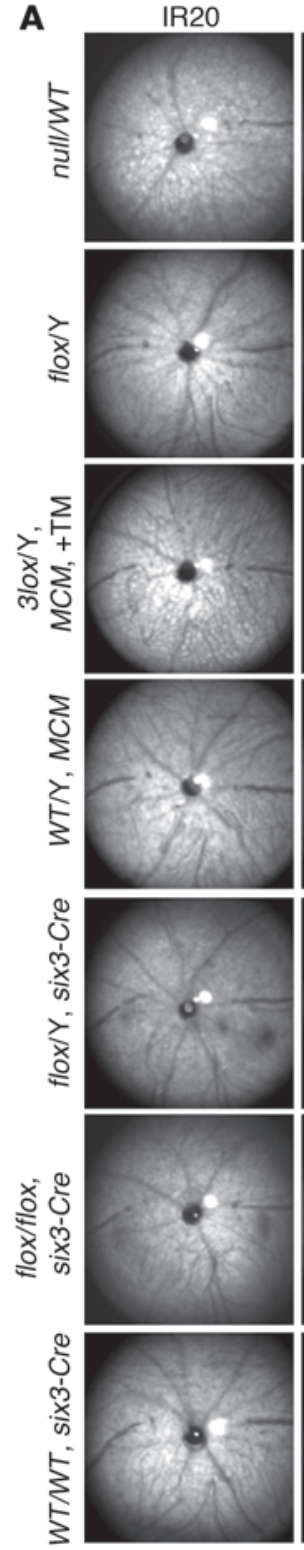

RF20
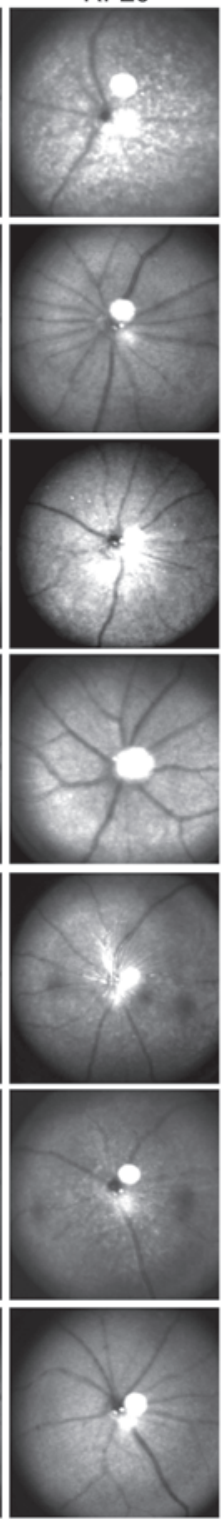

RF10
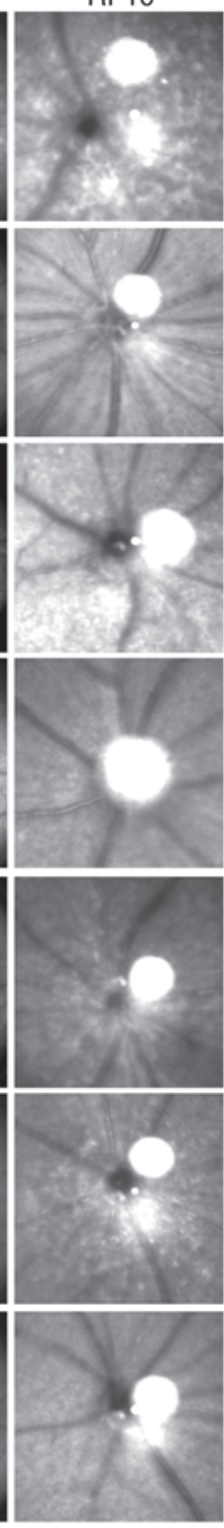

AF10
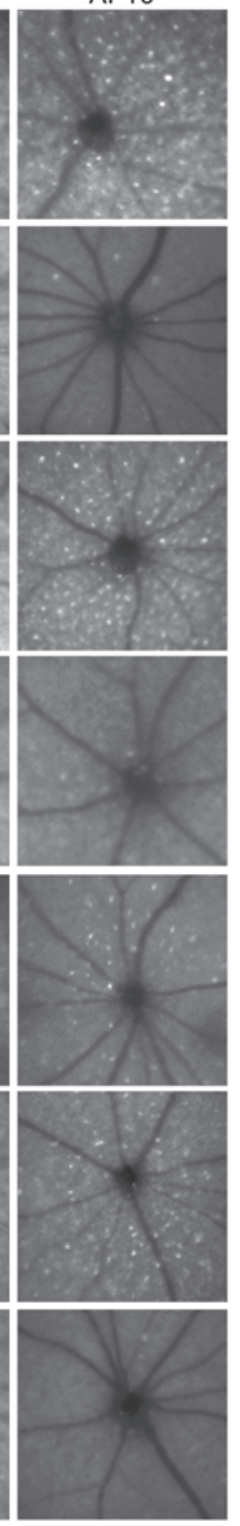

\section{B}
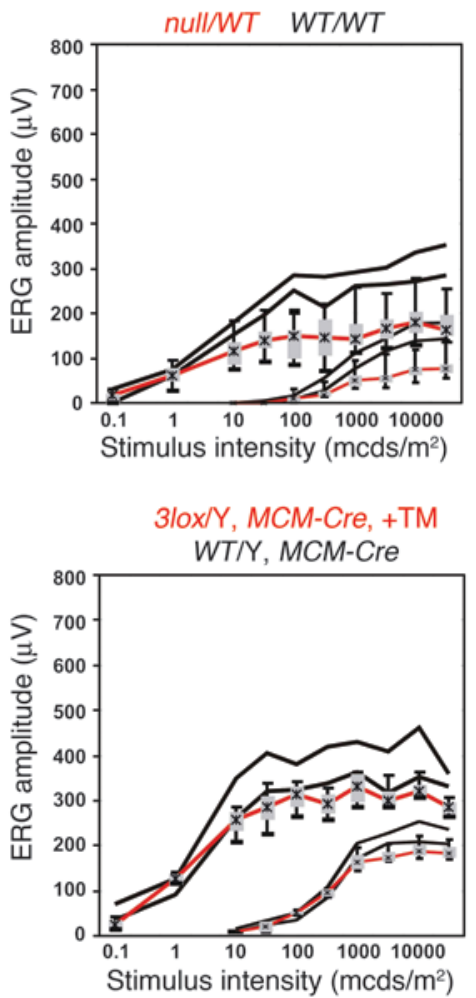

six3-Cre, flox/Y, and flox/flox flox/Y, flox/flox

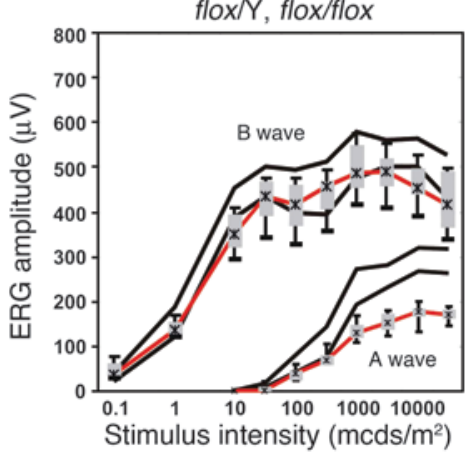

Figure 3

Ophthalmic and electroretinographic analysis of Chm mutant mouse eyes. (A) Retinal SLO images from representative Chm mutant animals. The Chm alleles, MerCreMer (MCM) transgene, six3-Cre transgene, and TM induction (+TM) are indicated to the left of each set of panels. An equipment setting of $20^{\circ}$ was used for a full view and of $10^{\circ}$ for a more detailed view. The wavelengths shown are infrared $\left(890 \mathrm{~nm}\right.$ and $20^{\circ}$, IR20), green (514 nm and $20^{\circ}$ or $10^{\circ}, \mathrm{RF} 20$ and RF10), and blue autofluorescence (488 nm with barrier at $500 \mathrm{~nm}$ and $\left.10^{\circ}, \mathrm{AF} 10\right)$. (B) Electroretinographic analysis showing a and $b$ waves for the indicated strains of mice. Box and whisker plot of mutant mouse data (red) (whiskers $5 \%$ and $95 \%$ quantile, box $25-75 \%$ quantile, asterisk median) in comparison with respective control mouse data (black) (5\% and $95 \%$ quantiles indicated by the lower and upper black lines, respectively, delimiting a $90 \%$ normal range). mcds, millicandela seconds.

RPE pathology was detected as irregular areas of abnormal depigmentation distributed throughout the whole retina (Figure 3A, IR and RF channels). Small patches of hypopigmentation were apparent on examination of 1-month-old animals, and these were noticeably expanded by 2 months of age. In 4-month-old and older animals, these areas became confluent, forming islands interspersed with intact retina (Figure $3 \mathrm{~A}$ and Supplemental Figure 1). Whitish autofluorescent flecks (Figure 3A, AF channel) were observed instead of the normal uniform appearance. The more irregular, dim variety of fleck may represent areas of RPE depigmentation, while the dotlike, round type possibly represents intraretinal changes (usually lipid accumulation) found in cases where outer-segment material is not digested normally by the RPE (as observed in rosettes or acute light damage).

Electroretinographic analysis showed a clear reduction of the scotopic (dark-adapted) a and b wave amplitude (Figure 3B), which is in agreement with previously published results and our own unpublished observations in CHM patients $(19,26)$. In par- 


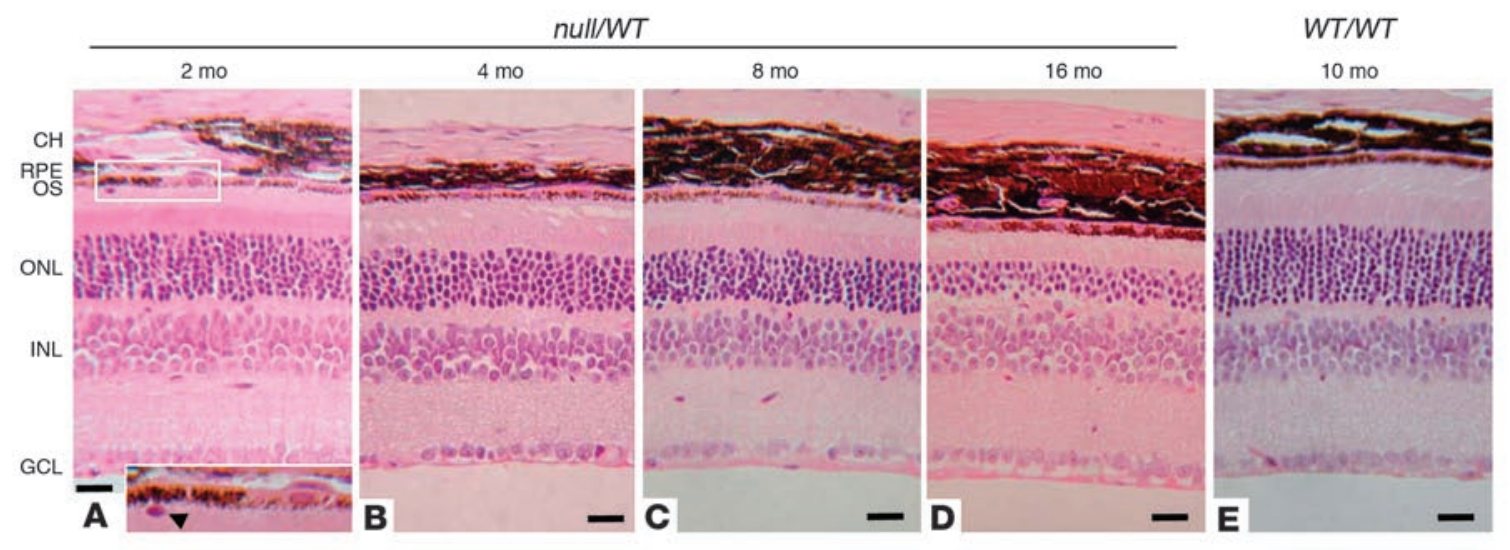

null/WT, 8 mo
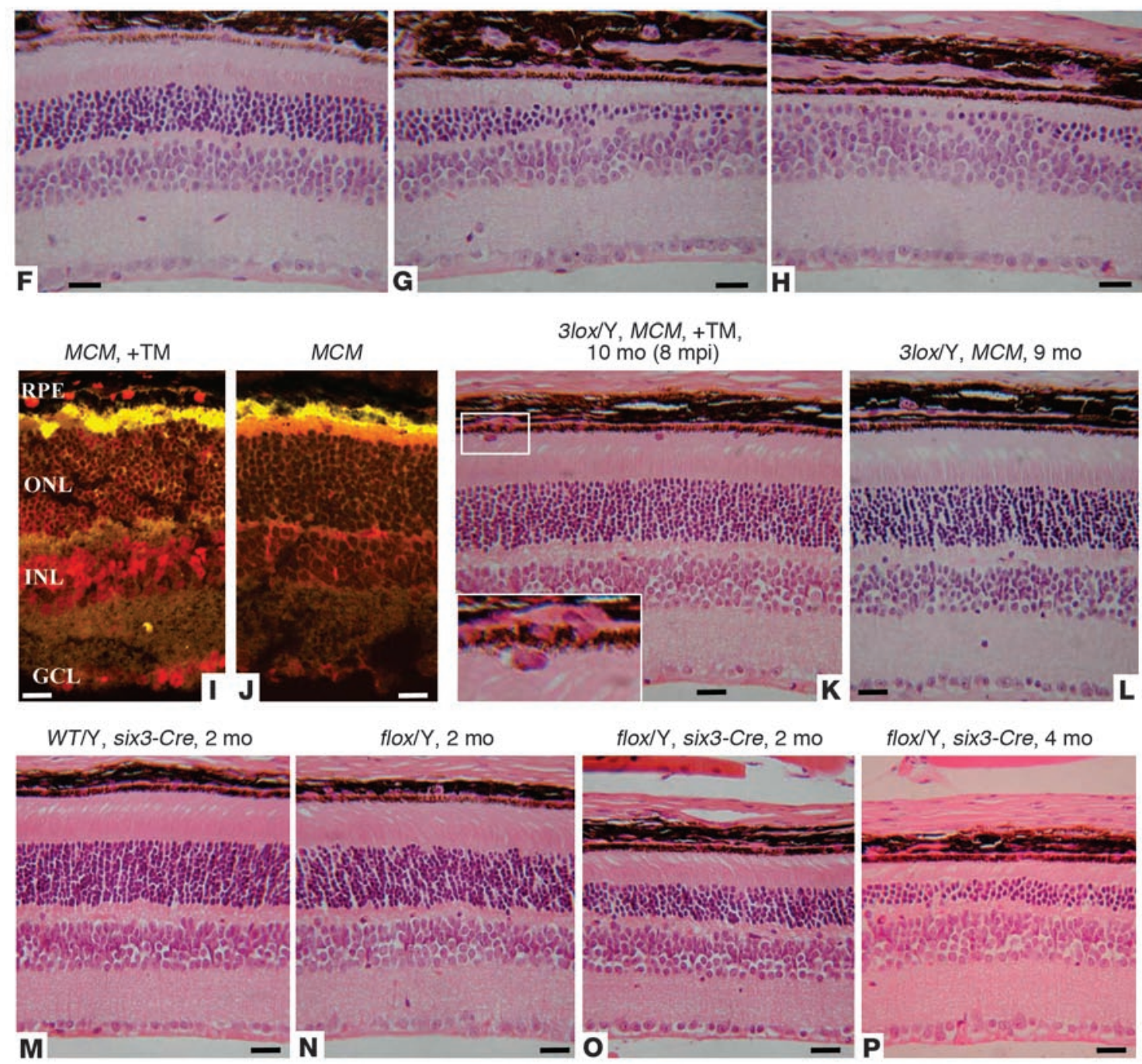

Figure 4

Histological analysis of sections of eyes of Chm mutant mice. (A-H) Chm null/wT mice at $2(\mathbf{A}), 4(\mathbf{B}), 8(\mathbf{C}$ and $\mathbf{F}-\mathbf{H})$, and 16 (D) months, and $\mathrm{Chm}^{W T / W T}$ mice at 10 months (E). (I and $\left.\mathbf{J}\right)$ Detection of Cre protein (red) in TM-induced (+TM) (I) and non-TM-induced (J) mice carrying the MerCreMer transgene (MCM) with a Cre-specific antibody. (K and L) Histological analysis of TM-induced (+TM) (K) and non-TM-induced (L) $\mathrm{Chm}^{3 / 0 x} / \mathrm{Y}$ mice carrying the MerCreMer transgene (MCM) at 9-10 months. Analysis was carried out 8 months after TM injection (8 mpi). (M-P) Two-month-old $C h m^{W T} / Y(\mathbf{M})$ and $C h m^{f l o x} / Y(N)$ mice, and $C h m^{f l o x} / Y$ six3-Cre+ mice at $2(\mathbf{O})$ and $4(\mathbf{P})$ months. $\mathrm{CH}$, choroid; RPE, retinal pigment epithelium; OS, photoreceptor outer segments; ONL, outer nuclear layer; INL, inner nuclear layer; GCL, ganglion cell layer. Scale bars: 20 um. Magnification, $\times 230$ (A, inset), $\times 450$ (K, inset). 


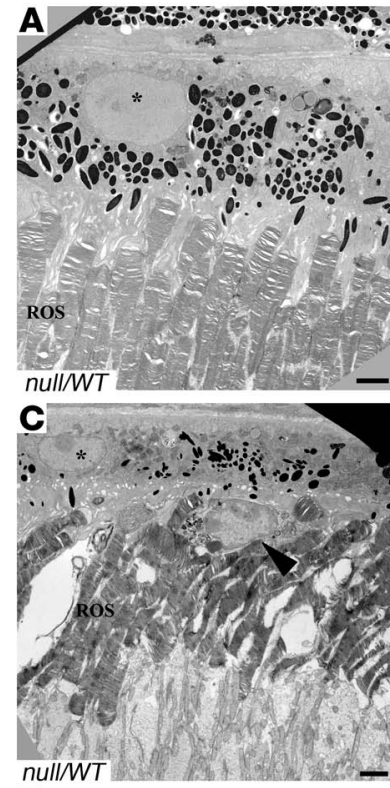

$\mathbf{E}$



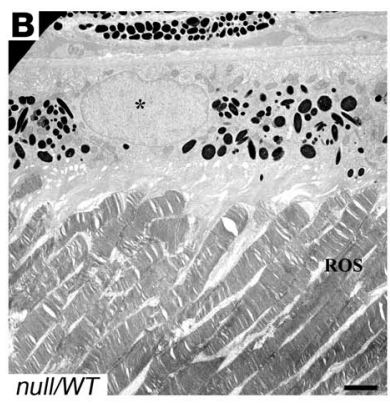

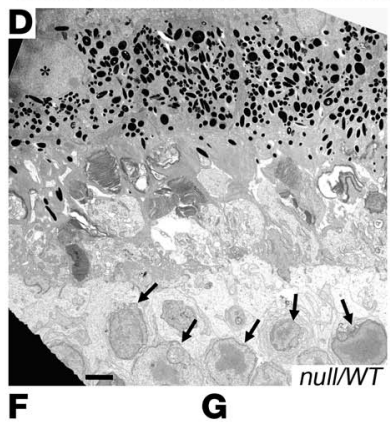

flox/Y, six3-Cre WT/Y, six3-Cre

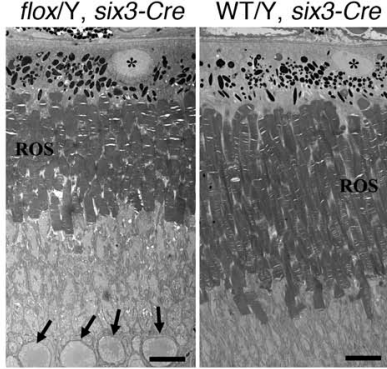

Figure 5

Electron microscopic analysis of $\mathrm{Chm}$ mutant retinas. (A-D) Different sections from the eye of a 9-month-old $C h m^{\text {null/WT }}$ female mouse. (E) A TM-induced $\mathrm{Chm}^{3 / 0 x} / \mathrm{Y}$ MerCreMer mouse. (F) A Chm ${ }^{\text {flox/Y }} / \mathrm{Y}$ six3-Cre mouse. (G) A Chm ${ }^{W T} / Y$ six3-Cre mouse. RPE nuclei are marked by asterisks. Abnormal cells above the RPE are shown by arrowheads in $\mathbf{C}$ and $\mathbf{E}$. Nuclei of cells from the ONL are marked by arrows in $\mathbf{D}$ and F. Scale bars: $2 \mu \mathrm{m}(\mathbf{A}-\mathbf{E})$ or $7 \mu \mathrm{m}(\mathbf{F}$ and $\mathbf{G})$.

ticular, the comparison between the electroretinogram (ERG) in heterozygous females and that in unaffected males (both from the $\mathrm{F}_{1}$ generation) in the study by van den Hurk et al. provided evidence for a substantial amplitude reduction in the heterozygotes, similar to what we describe here in $C h m^{\text {null/WT}}$ animals.

Histological analysis reveals a progressive and severe retinal degeneration. Histological analysis showed that the photoreceptors underwent late-onset progressive degeneration in adult $\mathrm{Chm}^{\text {null/WT }}$ mice, reminiscent of that in CHM patients. In 2-month-old female carriers (Figure 4A), the photoreceptor layer (outer nuclear layer, ONL) was slightly reduced in thickness (8-10 instead of 10-12 nuclei thick). At 4 months, the depth of the ONL was on average 7-8 nuclei (Figure 4B). In 8-month-old and older animals, most of the ONL consisted of 5-6 nuclei (Figure 4, C and D), although there were focal areas of severe degeneration where only 1-2 nuclei or no photoreceptor cells were left (Figure 4, G and H) as well as mildly affected areas (Figure 4F). In addition, patchy depigmentation of the RPE was observed in most sections (Figure 4A, inset), and we noticed frequent abnormal cells next to the RPE (Figure 4A, inset, arrow-

head; and Figure 4, G and K). Such cells were only occasionally seen in wild-type and other control mice, including $\mathrm{Chm}^{3 l o x}$ mice. The abnormal cells possessed very limited cytoplasm, and the nuclei were similar to those of the RPE in shape and size. We also noted that the progressive degeneration of RPE cells resulted in thinning of the RPE layer, a characteristic of human CHM disease (27).

A more detailed analysis of the retina of a 9-month-old Chm mull/WT $^{\text {ind }}$ female was performed by electron microscopy (Figure 5, A-D). Figure 5A displays an example of an area where the RPE and rod outer segments (ROSs) are apparently normal. Figure 5B depicts a region where the RPE is slightly depigmented with redistribution of pigment granules to the basal area of the cell, similar to that found in Rab27a-deficient (ashen) and myosin VIIa-deficient (shaker-1) mice (28), but the ROSs have wild-type morphology. Another region (Figure 5C) shows a severely depigmented RPE cell (asterisk) next to shortened and structurally altered ROSs. An abnormal cell next to the RPE is highlighted by the arrowhead. In Figure 5D, pigmented RPE cells are adjacent to severely degenerated photoreceptors. Most of the ROSs and the inner segments have disappeared, resulting in photoreceptor nuclei (marked by arrows) approaching the RPE. These data suggest that there is no direct correlation between degeneration of the photoreceptors and depigmentation of the RPE.

Early developmental defects. The early development of the retina in Chm ${ }^{\text {null/WT }}$ and Chm $m^{\text {null }+N e o / W T}$ mice was also affected. At the earliest stage studied, P7, patchy depigmentation of the RPE was observed but other layers appeared normal (Figure 6A, inset). Some cells were devoid of pigment granules, while others looked indistinguishable from wild type. Later, at P15 (Figure 6, C and D) and P17 (Figure 6, E and F), the ONL was reduced in thickness by approximately $20-25 \%$. There was also noticeable delay in the development of the outer segments, which were $50 \%$ shorter than wild type at P15 and P17, while the length of the inner segments was similar to wild type (Figure 6, C-F). Although development of photoreceptors was affected at P15 and P17, an apparently normal photoreceptor layer, with a thickness of 10-12 nuclei and normal inner and outer segments, was present in 1-month-old mice (Figure 5, G and $\mathrm{H}$ ). These data suggest that $\mathrm{Chm}$ knockout causes early RPE defects and delays development of the photoreceptors, although the overall appearance at 1 month is normal.

Tissue-restricted knockout of Chm shows cell-autonomous degeneration. Electron microscopy analysis of the retina of a Chm $\mathrm{Cull}^{\mathrm{w} T}$ mouse had suggested that the photoreceptor and RPE layers degenerate independently (Figure 5, A-D). To further investigate this important issue, we attempted to generate tissue-restricted Chm knockouts. Firstly, we analyzed $\mathrm{MerCreMer}{ }^{+} \mathrm{Chm}^{3 l o x} / Y$ mice, which were induced with TM at 6-8 weeks of age. Immunostaining using Cre antibody on TM-induced and non-TM-induced $\mathrm{MerCreMer}^{+}$mice showed that TM treatment induced translocation of Cre into the nucleus in all cell layers of the eye except the photoreceptors (Figure 4, I and J). Histological examination showed no obvious photoreceptor degeneration up to 14 months after induction (Figure $4, \mathrm{~K}$ and $\mathrm{L}$ ), consistent with the lack of Cre activation in these cells. However, there were frequent abnormal cells adjacent to the RPE layer in TM-induced $\mathrm{Chm}^{3 l o x} / \mathrm{Y}$ (and $\mathrm{Chm}^{3 l o x / 3 l o x}$ ) $\mathrm{MerCreMer}^{+}$mice (Figure 4K, inset), similar to those detected in Chm ${ }^{\text {null } / W T}$ mice. Electron microscopy showed remnants of melanosomes in these cells, again suggesting that they probably originate in the RPE (Figure 5E). SLO examination of TM-induced $\mathrm{MerCreMer}^{+} \mathrm{Chm}^{3 l o x} / \mathrm{Y}$ mice showed abnormal pigmentation at the RPE level, confirming RPE defects similar to those in Chm ${ }^{\text {mull/WT}}$ mice but at a slightly reduced 


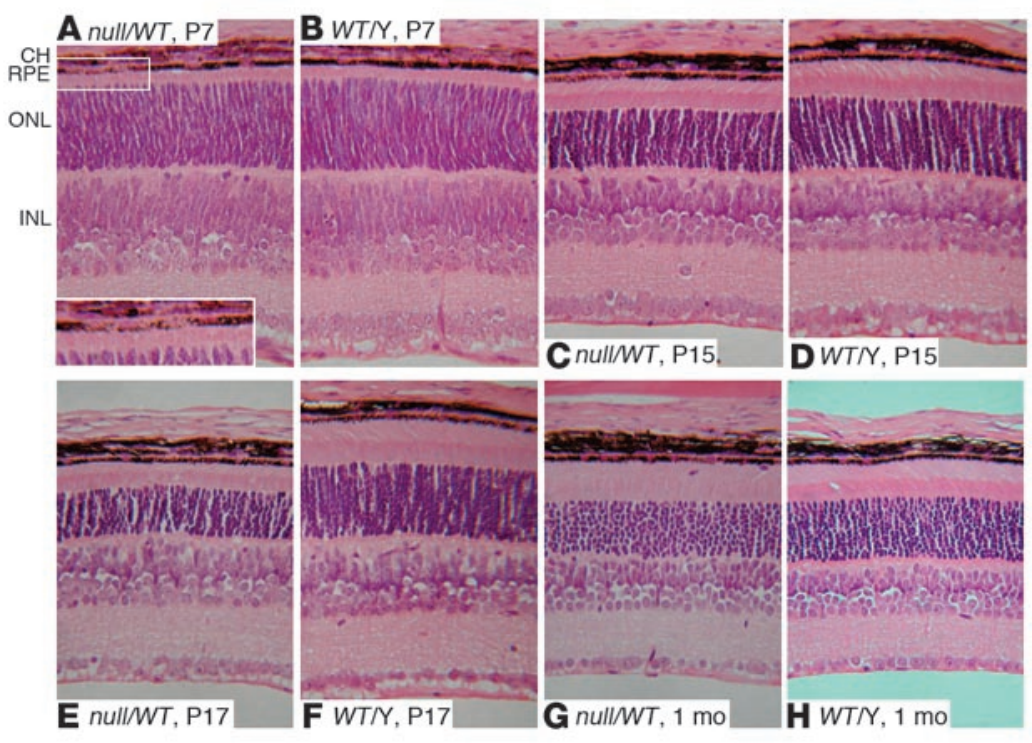

\section{Figure 6}

Histological analysis of $\mathrm{Chm}$ mutant retinas during development. Sections from $\mathrm{Chm}^{\text {null/WT}}$ and $\mathrm{Chm} \mathrm{m}^{\mathrm{WT}} / \mathrm{Y}$ littermates at P7, P15, and P17 and at 1 month as indicated for each panel. Magnification, $\times 125(\mathbf{A}-\mathbf{H}), \times 210(\mathbf{A}$, inset).

ed that other Rabs may also be unprenylated (Figure 2D). We further investigated the pattern of unprenylated Rabs in the eyes of the different tissue-restricted mouse models using in vitro prenylation reactions on cytosolic fractions of eye lysates (Figure 2E). The pattern of bands in eye extracts derived from TM-induced $\mathrm{Chm}^{3 l o x} / \mathrm{Y}$ (or Chm flox $/ \mathrm{Y}$ ) MerCreMer ${ }^{+}$mice was significantly different from that for $\mathrm{Chm}^{\text {flox }} / \mathrm{Y}$ six $3-\mathrm{Cre}^{+}$mice. This suggests that there are different subsets of Rabs in photoreceptors and in the RPE, which are underprenylated in the absence of Rep1. This biochemical analysis further suggests that CHM defects are triggered independently in the RPE and photoreceptors. level (Figure 3A), and tiny autofluorescent specks, which indicated localized intraretinal irregularities. ERG recordings demonstrated a and $b$ waves at the lower margin of the normal limits over the entire range of stimuli (Figure 3B), suggesting no significant and/or generalized abnormalities of photoreceptor cells. Thus, our analysis of TM-induced $\mathrm{Chm}^{3 l o x} / \mathrm{Y}$ (and $\mathrm{Chm}^{3 l o x / 3 l o x}$ ) MerCreMer ${ }^{+}$mice shows that the RPE degenerates without major effects on photoreceptors.

$\mathrm{Chm}^{\text {flox }}$ mice were also bred with mice carrying a six3-Cre transgene (29). Six 3 is a homeobox transcription factor that is expressed in the neuroretina during eye development $(30,31)$. Consistent with this, we did not observe depigmentation of the RPE nor abnormal cells next to the RPE in $\mathrm{Chm}^{\text {flox }} / \mathrm{Y}$ (or $C h m^{\text {flox/flox}}$ ) mice carrying the six3-Cre transgene (Figure 4, O and $\mathrm{P}$ ). Nevertheless, prominent degeneration of photoreceptors was observed. The ONL of six3-

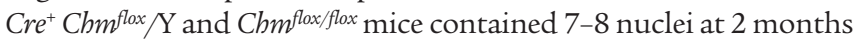
(Figure 4O) and 4-5 nuclei at 4 months (Figure 4P). Additionally, electron microscopic examination showed that photoreceptor outer segments exhibited significantly shorter average length, while RPE cells looked normal (Figure 5, F and G). A selective decrease in the a wave of the scotopic single-flash ERG in six3-Cre $e^{+} \mathrm{Chm}^{\text {flox }} / \mathrm{Y}$ and $\mathrm{Chm}^{f l o x / f l o x}$ mice indicated a generalized defective function of photoreceptor cells (Figure 3B and Supplemental Figure 2). The loss of the a wave component apparent in Figure $3 \mathrm{~B}$ is further characterized in Supplemental Figure 2. In particular, the A/B ratio was found to be significantly lower in the mutant mice $(P<0.0013)$, and there was no overlap between the ranges of individual traces from both groups at high-intensity stimuli. SLO imaging revealed a substantial number of autofluorescent intraretinal specks in six $3-\mathrm{Cre}^{+} \mathrm{Chm}^{\text {flox }} / \mathrm{Y}$ (and $\mathrm{Chm}^{\text {flox/flox}}$ ) mice (Figure 3A), consistent with photoreceptor damage. The visible patchy RPE layer abnormalities were, at that stage, rather mild and bore close resemblance to the secondary changes observed in mouse models subsequent to the total loss of photoreceptor cells (32). Presumably, these changes correspond to regions with severely reduced photoreceptor layer. We conclude that $\mathrm{Chm}^{\text {flox }} / \mathrm{Y}$ six $3-\mathrm{Cr} e^{+}$ (and $\mathrm{Chm}^{\text {flox/flox }}$ six $3-\mathrm{Cr} \mathrm{e}^{+}$) mice develop severe photoreceptor degeneration, initially without significant effects on the RPE.

Involvement of different Rabs in degeneration of the photoreceptors and the $R P E$. Although the prenylation of Rab27a was specifically affected in $\mathrm{Chm}^{\text {mull } / W T}$ tissues (Figure 2A), in vitro prenylation reactions suggest-

\section{Discussion}

The purpose of this study was to create a mouse model of CHM, a degenerative eye disorder affecting the photoreceptors, RPE, and the choroid, which is caused by loss of function of the CHM gene. Using a conditional approach, we were able to generate a viable colony of mice producing female carriers of a Chm-null allele that develop retinal disease with characteristics similar to those of human CHM. Our analysis of disease progression suggests that the degenerative process develops independently in 2 key affected layers, photoreceptor and RPE cells.

The interdependence between photoreceptors and RPE is a critical issue in unraveling the pathogenic mechanisms underlying retinal degenerations. Several genetic photoreceptor degenerations are caused by mutations in genes expressed by the RPE, such as RPE65, MERTK, RDH5, RGR, and $\operatorname{LRAT}(1,3)$. Conversely, a photoreceptor gene $(A B C A 4)$ is thought to cause primarily RPE degeneration in one form of Stargardt disease $(1,3)$. In that case, the RPE degeneration is then thought to induce secondary photoreceptor degeneration. In this study, we observed clear evidence of RPE degeneration without noticeable effects on photoreceptor number in TM-induced $\mathrm{MerCreMer}^{+} \mathrm{Chm}^{3 l o x} / \mathrm{Y}$ mice, as late as 14 months after induction. Our data indicate that RPE degeneration does not lead necessarily to photoreceptor degeneration, raising the possibility that most retinal degenerative processes involve cellautonomous defects and that secondary effects are mostly exerted in the advanced stages of disease owing to the many supporting functions RPE cells perform for photoreceptors.

Our data support the idea that defective prenylation of a subset of Rabs underlies the CHM phenotype or is at least partially responsible for its appearance $(17,18)$. We observed a dramatically different pattern of nonprenylated Rabs in the 2 tissue-restricted KO models, suggesting that a different subset of Rabs might be responsible in each cell type for the defects that ultimately lead to degeneration. We find particularly interesting the observation that 1 major Rab may be dysfunctional in $\mathrm{Chm}^{f l o x} / \mathrm{Y} s i x 3-\mathrm{Cre}^{+}$mice, where severe photoreceptor (but not RPE) degeneration is manifested. If photoreceptor degeneration is triggered by the dysfunction of this Rab, it must be performing a specific and important function. Future studies should aim to identify this photorecep- 
tor Rab and the RPE-specific Rabs affected, and to elucidate the important functions they play in these specialized cell types.

This work has important implications for the management and treatment of CHM patients. Our analysis of disease progression suggests that the degenerative process develops independently in the 2 key affected layers, photoreceptor and RPE cells, and ideally both cell types should be targeted. Nevertheless, restoration of Rep1 function in photoreceptors appears to be of critical importance, as these cells degenerate rapidly in the absence of RPE disease, as we observed in $\mathrm{Chm}^{\text {flox }} \mathrm{Y}$ six 3-Cre ${ }^{+}$mice. In addition, we note that the choroidal component of CHM remains mostly uncharacterized. We will begin specific studies to address this issue in our newly created lines.

In conclusion, a conditional knockout approach allowed us to overcome the breeding problems caused by the embryonic lethality of Chm-null alleles. Eye studies of heterozygous $\mathrm{Chm}^{\text {null/WT }}$ females showed features of progressive retinal degeneration similar to those associated with CHM in humans. Thus, the heterozygous Chm-null females are a valid model for CHM and will be used for further studies of the disease pathogenesis and for future gene therapy trials.

\section{Methods}

Generation of Chm $\mathrm{Chlox}^{3 l i c e}$. Thirty micrograms of targeting vector pTT55 was linearized with $X$ hoI endonuclease, purified by ethanol precipitation, and used for electroporation of $3 \times 10^{7}$ GSI- 1 cells, which were then cultured in the presence of G418 $(0.4 \mathrm{mg} / \mathrm{ml})$. Initial screening for 3 '-end integration events by Southern blotting with HindIII digestion and the 3 ' probe (Figure $1, \mathrm{~A}$ and $\mathrm{B}$ ) resulted in 17 positive clones out of 408 analyzed. Twelve of these 17 clones were further screened for correct integration at the $5^{\prime}$ end with Sst I digestion and the $5^{\prime}$ probe (Figure 1B), for the absence of multiple integrations of the vector using a Neo probe, and for the presence of the $5^{\prime}$-most loxP site. All 12 analyzed clones had correct integration at the $5^{\prime}$ end, and no multiple integrations. The $5^{\prime}$-most loxP site was present in only 10 out of 12 clones. Blastocyst injections with ES cell line 92 (Figure $1 \mathrm{~B}$ ) resulted in 3 chimeric males, of which 1 successfully transmitted the $\mathrm{Cbm}^{3 l o x}$ allele through the germ line. These crosses produced several positive $\mathrm{F}_{2}$ offspring, both male and female, suggesting that the modified $\mathrm{Chm}^{3 l o x}$ allele is transmitted normally through the germ line. Routine genotyping of mice was performed by PCR. Primers H7 (5'-AGAGTATCTCAGCAGTAGCTCTCC) and H9 (5'-CCAGAGAACACTGAGGGTTAGAGC) allowed identification of $C h m^{W T}, C h m^{f l o x}$, and $C h m^{\text {null }}$ alleles. The sizes of the resultant PCR products were: $780 \mathrm{bp}\left(\mathrm{Chm^{WT }}\right), 860 \mathrm{bp}\left(C h m^{f l o x}\right)$, and $330 \mathrm{bp}\left(C h m^{\text {null }}\right)$. To confirm the presence of $C h m^{3 l o x}$ and $C h m^{\text {null }+ \text { Neo }}$ alleles, 2 additional PCRs were used. Spectinomycin-specific primers Spec4 (5'GATGTTATGGAGCAGCAACG) and Spec5 (5'-CTTCTTGTCCAAGATAAGCC) allowed confirmation of the presence of a neomycin-spectinomycin resistance cassette, and a 770-bp PCR product indicated the presence of either an $\mathrm{Chm}^{3 l o x}$ or $\mathrm{Chm}^{\text {null }+ \text { Neo }}$ allele. PCR analysis using primers $\mathrm{H} 7$ and $\mathrm{H} 8$ (5'-CCAACAGTGTGCCAGCTTCAAAGG) resulted in either a 420-bp PCR product (for $\mathrm{Chm}^{3 l o x}$ and $\mathrm{Chm} m^{f l o x}$ ) or a 370-bp PCR product (for $\mathrm{Chm}{ }^{W T}$ ), while for $\mathrm{Chm}^{\text {null }+ \text { Neo }}$ and $\mathrm{Chm}^{\text {null }}$ alleles there was no amplification. Cre-specific primers Cre1 (5'-TCCCGCAGAACCTGAAGATGTTC) and Cre2 (5'GGATCATCAGCTACACCAGAGACG) were used to screen for MerCreMer and six3-Cre transgenes, the size of the product was $510 \mathrm{bp}$. Cycling conditions were $94^{\circ} \mathrm{C}$ for $3 \mathrm{~min}$, followed by 32 cycles of: $94^{\circ} \mathrm{C}$ for $40 \mathrm{~s}, 62^{\circ} \mathrm{C}$ for $40 \mathrm{~s}, 72^{\circ} \mathrm{C}$ for $1 \mathrm{~min}$, and $72^{\circ} \mathrm{C}$ for $10 \mathrm{~min}$.

Mouse strains. Mice were bred and maintained in the Imperial College animal facility in accordance with the rules and regulations of the Home Office on project licenses 70/5056 and 70/6176. The transgenic mouse line $\beta$ MCM70 carries a TM-inducible MerCreMer transgene under control of the CMV-enhanced chicken $\beta$-actin promoter (pCAGGS) (22,
33). TM (Sigma-Aldrich) was administered to 6- to 8-week-old mice by 5 i.p. injections on 5 consecutive days ( $1 \mathrm{mg}$ of TM per injection). The transgenic six3-Cre mice carry a Cre-recombinase gene with the nuclear localization signal of the SV40-T gene inserted into the first coding exon of the mouse six3 gene (29).

Biochemical studies. Protocols for immunoblotting and subcellular fractionation have been described previously (34). The antibodies used were polyclonal anti-Rep antibody J905, which recognizes Rep1 and Rep2 proteins (dilution 1:500) (35); monoclonal anti-rat Rab27a antibody 4B12 (dilution 1:10,000) (36); polyclonal anti-calnexin antibody (Stressgen Bioreagents Corp.; dilution 1:5,000); monoclonal anti-Rep8 antibody (BD Transduction Laboratories, BD Biosciences - Pharmingen; dilution 1:1,000); and monoclonal anti-Rep11 antibody (BD Transduction Laboratories, BD Biosciences - Pharmingen; dilution 1:1,000).

Morphological studies. Mouse eyes for histology were fixed (2\% paraformaldehyde, $2.5 \%$ glutaraldehyde, $0.1 \mathrm{M}$ cacodylate buffer) for 1 hour. Samples were embedded in paraffin wax, and sections were cut at $4 \mu \mathrm{m}$ thickness and stained with H\&E. Electron microscopy was performed as described previously (28).

Electrophysiological studies. ERGs and SLO were obtained in dark-adapted mice according to previously reported procedures $(37,38)$. The ERG equipment consisted of a Ganzfeld bowl, a direct current amplifier, and a PCbased control and recording unit (Multiliner Vision; VIASYS Healthcare). SLO was performed with a Heidelberg Retina Angiograph (Heidelberg Engineering), a confocal scanning-laser ophthalmoscope. Laser wavelengths used for fundus visualization were $835 \mathrm{~nm}$ (infrared channel), $514 \mathrm{~nm}$ (red-free channel), and $488 \mathrm{~nm}$ (for autofluorescent images). The following types of mice and numbers of eyes were analyzed with ERG and SLO for this study: Chm $m^{\text {null/WT }}$ (10 eyes), Chm ${ }^{W T / W T}$ (4 eyes), Chm ${ }^{W T} / \mathrm{Y}$ MerCreMer (4 eyes), Chm ${ }^{3 l o x} / \mathrm{Y}$ MerCreMer TM+ (4 eyes), Chm $^{W T / W T}$ six $3-\mathrm{Cre}^{+}$ (2 eyes), Chm ${ }^{f l o x} / \mathrm{Y}$ (4 eyes), Chm floxfflox (2 eyes), Chm flox $/ \mathrm{Y} s i x 3-C r e^{+}$( 4 eyes), and Chm flox/flox six $3-\mathrm{Cre}^{+}$(6 eyes).

Statistics. To quantify the loss of the a wave relative to the $\mathrm{b}$ wave of the ERG, a/b ratios were calculated as shown in Supplemental Figure 2. The evaluation was based on the data obtained with the maximum stimulus intensity of 25 candela seconds $/ \mathrm{m}^{2}$. Groups were compared using a 2-sample $t$ test with assuming unequal variances. A $P$ value less than or equal to 0.05 was considered statistically significant.

\section{Acknowledgments}

This paper is dedicated to all choroideremia patients. We would like to thank G. Oliver, Y. Furuta, and N. Tekki-Kessaris for the six3-Cre transgenic line; B. Wanner, P. Ioannou, B. Bigger, and O. Tolmachov for donation of reagents; M. Gegg for help with analysis of mouse Chm bacterial artificial chromosomes; L. Lawrence for help with histology; R. Panford-Walsh for the preliminary ERG and SLO experiments; and C. Gregory-Evans for valuable comments and advice. This work was supported by the Foundation Fighting Blindness (USA), the Choroideremia Research Foundation, the German Research Council (DFG Se837/1-2 and Se837/4-1), the University of Tuebingen (fortuene grant 1173), and an anonymous private donor.

Received for publication August 18, 2005, and accepted in revised form November 1, 2005.

Address correspondence to: Miguel C. Seabra, Molecular and Cellular Medicine, Division of Biomedical Sciences, Sir Alexander Fleming Building, Imperial College London, Exhibition Road, London SW72AZ, United Kingdom. Phone: 44-20-7594-3024; Fax: 44-20-7594-3015; E-mail: m.seabra@imperial.ac.uk. 
1. Pacione, L.R., Szego, M.J., Ikeda, S., Nishina, P.M., and McInnes, R.R. 2003. Progress toward understanding the genetic and biochemical mechanisms of inherited photoreceptor degenerations. Annu. Rev. Neurosci. 26:657-700.

2. Stone, E.M., Sheffield, V.C., and Hageman, G.S. 2001. Molecular genetics of age-related macular degeneration. Hum. Mol. Genet. 10:2285-2292.

3. Strauss, O. 2005. The retinal pigment epithelium in visual function. Physiol. Rev. 85:845-881.

4. Heckenlively, J.R., and Bird, A.J. 1988. Choroideremia. In Retinitis pigmentosa. J.R. Heckenlively, editor. Lippincott Williams \& Wilkins. Philadelphia, Pennsylvania, USA. 176-187.

5. Cremers, F.P.M. 1995. Choroideremia. In The metabolic and molecular bases of inherited disease. C.B. Scriver, A.L. Beaudet, W.S. Sly, and D. Valle, editors. McGrawHill. New York, New York, USA. 4311-4324.

6. MacDonald, I.M., Sereda, C., McTaggart, K., and Mah, D. 2004. Choroideremia gene testing. Expert Rev. Mol. Diagn. 4:478-484.

7. Cremers, F.P., van de Pol, D.J., van Kerkhoff, L.P., Wieringa, B., and Ropers, H.H. 1990. Cloning of a gene that is rearranged in patients with choroideraemia. Nature. 347:674-677.

8. Seabra, M.C., Brown, M.S., Slaughter, C.A., Sudhof, T.C., and Goldstein, J.L. 1992. Purification of component A of Rab geranylgeranyl transferase: possible identity with the choroideremia gene product. Cell. 70:1049-1057.

9. Andres, D.A., et al. 1993. cDNA cloning of component A of Rab geranylgeranyl transferase and demonstration of its role as a Rab escort protein. Cell. 73:1091-1099.

10. Zerial, M., and McBride, H. 2001. Rab proteins as membrane organizers. Nat. Rev. Mol. Cell Biol. 2:107-117.

11. Seabra, M.C., Mules, E.H., and Hume, A.N. 2002. Rab GTPases, intracellular traffic and disease. Trends Mol. Med. 8:23-30.

12. Seabra, M.C. 2000. Biochemistry of Rab geranylgeranyl transferase. In Lipid modifications of proteins. F. Tamanoi and D. Sigman, editors. Academic Press. New York, New York, USA. 131-154.

13. Casey, P.J., and Seabra, M.C. 1996. Protein prenyltransferases. J. Biol. Chem. 271:5289-5292.
14. Seabra, M.C., and Wasmeier, C. 2004. Controlling the location and activation of Rab GTPases. Curr. Opin. Cell Biol. 16:451-457.

15. Pereira-Leal, J.B., and Seabra, M.C. 2000. The mammalian Rab family of small GTPases: definition of family and subfamily sequence motifs suggests a mechanism for functional specificity in the Ras superfamily. J. Mol. Biol. 301:1077-1087.

16. Cremers, F.P., Armstrong, S.A., Seabra, M.C., Brown, M.S., and Goldstein, J.L. 1994. REP-2, a Rab escort protein encoded by the choroideremia-like gene. J. Biol. Chem. 269:2111-2117.

17. Seabra, M.C. 1996. New insights into the pathogenesis of choroideremia: a tale of two REPs. Ophthal mic Genet. 17:43-46.

18. Seabra, M.C., Ho, Y.K., and Anant, J.S. 1995. Deficient geranylgeranylation of Ram/Rab27 in choroideremia. J. Biol. Chem. 270:24420-24427.

19. van den Hurk, J.A., et al. 1997. Mouse choroideremia gene mutation causes photoreceptor cell degeneration and is not transmitted through the female germline. Hum. Mol. Genet. 6:851-858.

20. Shi, W., et al. 2004. Choroideremia gene product affects trophoblast development and vascularization in mouse extra-embryonic tissues. Dev. Biol. 272:53-65.

21. Starr, C.J., Kappler, J.A., Chan, D.K., Kollmar, R., and Hudspeth, A.J. 2004. Mutation of the zebrafish choroideremia gene encoding Rab escort protein 1 devastates hair cells. Proc. Natl. Acad. Sci.U. S. A. 101:2572-2577.

22. Zhang, Y., et al. 1996. Inducible site-directed recombination in mouse embryonic stem cells. Nucleic Acids Res. 24:543-548.

23. Detter, J.C., et al. 2000. Rab geranylgeranyl transferase alpha mutation in the gunmetal mouse reduces Rab prenylation and platelet synthesis. Proc. Natl. Acad. Sci. U. S. A. 97:4144-4149.

24. Meyers, E.N., Lewandoski, M., and Martin, G.R. 1998. An Fgf8 mutant allelic series generated by Cre- and Flp-mediated recombination. Nat. Genet. 18:136-141.

25. Nagy, A., et al. 1998. Dissecting the role of N-myc in development using a single targeting vector to generate a series of alleles. Curr. Biol. 8:661-664

26. Rudolph, G., et al. 2003. Phenotypic variability in three carriers from a family with choroideremia and a frameshift mutation 1388delCCinsG in the REP-1 gene. Ophthalmic Genet. 24:203-214.

27. Syed, N., et al. 2001. Evaluation of retinal photoreceptors and pigment epithelium in a female carrier of choroideremia. Ophthalmology. 108:711-720.

28. Futter, C.E., Ramalho, J.S., Jaissle, G.B., Seeliger, M.W and Seabra, M.C. 2004. The role of Rab27a in the regulation of melanosome distribution within retinal pigment epithelial cells. Mol. Biol. Cell. 15:2264-2275.

29. Furuta, Y., Lagutin, O., Hogan, B.L., and Oliver, G.C. 2000. Retina- and ventral forebrain-specific Cre recombinase activity in transgenic mice. Genesis. 26:130-132.

30. Bovolenta, P., Mallamaci, A., Puelles, L., and Boncinelli, E. 1998. Expression pattern of cSix3, a member of the Six/sine oculis family of transcription factors. Mech. Dev. 70:201-203.

31. Hsieh, Y.W., Zhang, X.M., Lin, E., Oliver, G., and Yang, X.J. 2002. The homeobox gene Six3 is a potential regulator of anterior segment formation in the chick eye. Dev. Biol. 248:265-280.

32. Jaissle, G.B., et al. 2004. In vivo and in vitro assessment of degenerative processes of retine, RPE, and vascular systems following the loss of photoreceptor cells in the rhodopsin knockout mouse. Invest. Ophthalmol. Vis. Sci. 45:E-Abstract5079.

33. Bugeon, L., et al. 2003. Inducible gene silencing in podocytes: a new tool for studying glomerular function. J. Am. Soc. Nephrol. 14:786-791.

34. Tolmachova, T., et al. 2004. A general role for Rab27a in secretory cells. Mol. Biol. Cell. 15:332-344.

35. Shen, F., and Seabra, M.C. 1996. Mechanism of digeranylgeranylation of Rab proteins. Formation of a complex between monogeranylgeranyl-Rab and Rab escort protein. J. Biol. Chem. 271:3692-3698.

36. Hume, A.N., et al. 2001. Rab27a regulates the peripheral distribution of melanosomes in melanocytes. J. Cell Biol. 152:795-808.

37. Seeliger, M.W., et al. 2001. New views on RPE65 deficiency: the rod system is the source of vision in a mouse model of Leber congenital amaurosis. Nat. Genet. 29:70-74.

38. Seeliger, M.W., et al. 2005. In vivo confocal imaging of the retina in animal models using scanning laser ophthalmoscopy. Vision Res. 45:3512-3519. 